\title{
Formulation Optimization Utilizing D-Optimal Experimental Design of Oral Capsules Containing Enteric-Coated Pellets of Lansoprazole and In Vivo Bioequivalence
}

\author{
Anh Quang Luong1,2, Thang Ngoc Vu33, Dang Hoa Nguyen4, Sultan M. Alshahrani5, \\ John Mark Christensen ${ }^{5 *}$, Chien Ngoc Nguyen ${ }^{*}$ \\ ${ }^{1}$ Department of Pharmacy, National Institute of Burns, Hanoi, Vietnam \\ ${ }^{2}$ Research and Training Center for Pharmacy, Military Medical University, Hanoi, Vietnam \\ ${ }^{3}$ Military Institute of Pharmaceutical Analysis and Research, Hanoi, Vietnam \\ ${ }^{4}$ Department of Pharmaceutics, Hanoi University of Pharmacy, Hanoi, Vietnam \\ ${ }^{5}$ Department of Pharmaceutical Sciences, College of Pharmacy, Oregon State University, Corvallis, Oregon, USA \\ ${ }^{6}$ National Institute of Pharmaceutical Technology, Hanoi University of Pharmacy, Hanoi, Vietnam \\ Email: ^jmark.christensen@oregonstate.edu, ^nguyenngocchien@yahoo.com, alshahrs@oregonstate.edu, \\ luongquanganh@vmmu.edu.vn,vuthangd8@gmail.com, hoayen09@yahoo.com
}

How to cite this paper: Luong, A.Q., Vu, T.N., Nguyen, D.H., Alshahrani, S.M., Christensen, J.M. and Nguyen, C.N. (2017) Formulation Optimization Utilizing D-Optimal Experimental Design of Oral Capsules Containing Enteric-Coated Pellets of Lansoprazole and In Vivo Bioequivalence. Pharmacology \& Pharmacy, 8, 153-171. https://doi.org/10.4236/pp.2017.85011

Received: April 8, 2017

Accepted: May 19, 2017

Published: May 22, 2017

Copyright $\odot 2017$ by authors and Scientific Research Publishing Inc. This work is licensed under the Creative Commons Attribution International License (CC BY 4.0).

http://creativecommons.org/licenses/by/4.0/

(c) (i) Open Access

\begin{abstract}
An optimized formulation of capsules containing Lansoprazole enteric-coated pellets using D-Optimal design with a polynomial statistical model were prepared by using Eudragit ${ }^{\oplus} \mathrm{L} 100$ as an enteric coated polymer to provide resistance to simulated gastric acid dissolution in buffer media. D-Optimal experimental design was used to determine the optimal level for three coating layers that were applied to formulate the enteric-coated pellets including a drug loading layer, a sub-coating, and an outer enteric coating. Dissolution studies were performed on the prepared Lansoprazole capsules. Less than 5 percent of Lansoprazole was released in 60 minutes in an acidic dissolution medium $(\mathrm{pH}$ 1.2 ) and greater than 90 percent of active ingredient was released in the next 60 minutes in a buffer dissolution medium ( $\mathrm{pH}$ 6.8). The Lansoprazole capsules were stable with no observable change in physico-chemical properties in accelerated and normal storage conditions for 6 and 18 months, respectively. The pharmacokinetic parameters $\mathrm{C}_{\max }, \mathrm{T}_{\max }, \mathrm{AUC}_{0-\mathfrak{t}}$ and $\mathrm{AUC}_{0-\infty}$ were determined after administration of the D-Optimal design optimized capsules of LPZ to healthy beagle dogs and were statistically compared to Gastevin ${ }^{\circledR}$ capsules as a reference (KRKA, Slovenia) using the non-compartmental method with the aid of WinNonlin 5.2 software. The analysis of variance showed that the two formulations did not demonstrate bioequivalence using a $90 \%$ confi-
\end{abstract}


dence interval range $(80 \%-120 \%)$ of $\mathrm{C}_{\max }, \mathrm{AUC}_{0-\mathrm{t}}$, and $\mathrm{AUC}_{0-\infty}$. No significant difference in $\mathrm{T}_{\max }$ was found at the 0.95 significance level using the Wilcoxon signed-rank test. D-Optimal Experimental Design provided definitive direction for an optimal formulation of capsules containing enteric-coated pellets of lansoprazole loaded within the coating of pellets that provided similar bioequivalence to Gastevin.

\section{Keywords}

Lansoprazole, D-Optimal, Pellets, Enteric-Coating, Pharmacokinetic Parameters

\section{Introduction}

Lansoprazole (LPZ), a proton pump inhibitor, is a lipophilic weak base with $\mathrm{pKa}$ values of 4.15 and 1.33 , where the $\mathrm{N}-\mathrm{H}$ proton in the benzimidazole ring is responsible for the acidity of the molecule ( $\mathrm{pKa} 8.84$ ). LPZ reduces gastric acidity, an important factor in healing acid-related disorders such as gastric ulcers, duodenal ulcers, and reflux esophagitis. It is used to treat gastro-oeesophageal reflux, ulcers, acid-related dyspepsia, and as an adjuvant in the eradication of Helicobacter pylori. It tends to relieve heartburn more effectively than omeprazole at therapeutic dosages [1]. However, degradation and the low water solubility of the lansoprazole lead to a reduction in its bioavailability when administrated orally. The degradation of lansoprazole in acidic medium is strongly $\mathrm{pH}$-dependent. The half-life of the LPZ in different $\mathrm{pH}$ 's varies dramatically: only 15 minutes at $\mathrm{pH} 2.0 ; 18$ hours at $\mathrm{pH} 6.0$, and up to 34 hours at $\mathrm{pH} 8.0$ [2]. The use of alkaline salts such as sodium carbonate, dibasic sodium phosphate, magnesium oxide, and magnesium carbonate have been indicated to increase the stability of LPZ, in which dibasic sodium phosphate is suggested as the most efficient stabilizer for protecting the drug against degradation [3]. Furthermore, an enteric-coated formulation is essential to protect the drug from the acidic environment of stomach.

The formulation challenge concerning a LPZ oral dosage form is the drug's poor water solubility producing poor absorption that results in low bioavailability. Efforts to improve the solubility of LPZ by utilizing a liquid solid technique, a solid dispersion system, and by spray drying were reported. The liquid solid technique use of Tween 80 as a carrier to increase wetting of the surface area available for enhanced LPZ's dissolution rate [4]. A solid dispersion system composed of LPZ, a surfactant such as Tween 80 , polyoxy 60 hydrogenated castor oil derivative (HCO-60), and PEG-8 caprylic/capric glycerides (Labrasol) presented the possibility of being a good method to improve the bioavailability of LPZ [5]. Additionally, utilizing the amorphous crystalline state of LPZ coupled with the presence of a hydrogen bond between LPZ and PVP K30 in a solid dispersion system markedly enhanced the dissolution rate more than $80 \%$ 
of drug within 30 minutes [6].

Pellets, multi-unit dosage forms, are easy to swallow while maintaining the merits of multiple units, bring about several therapeutic advantages including delayed of drug release, division of dose strength, and rapid distribution in the gastrointestinal tract when administered orally. In addition, a higher degree of flexibility in design and development during delivery of incompatible bioactive agents is also another pharmaceutical benefit of pellets [7].

The use of Eudragit L30D-55 as an enteric-coating polymer to formulate LPZ enteric-coated pellets, while at the same time using HPMC E5 for its role as a polymer in layering and sub-coating membrane for core pellets, the drug release from these enteric-coated pellets in $0.1 \mathrm{~N} \mathrm{HCl}$ and in phosphate buffer (pH 6.8) media was $0.71 \%$ and $97.87 \%$, respectively. The formulation was stable for 3 months at $40^{\circ} \mathrm{C} \pm 2{ }^{\circ} \mathrm{C} / 75 \% \mathrm{RH} \pm 5 \%$ [8]. In another study, three coating materials were used with manitol for the first layer, PVP K30 for the second layer, and HPMCP for the final layer in capsules of LPZ-modified release pellets to investigate controlled release properties of hypromellose phthalate by avoiding the gastric release of LPZ. The results showed that the drug release from capsules in an acidic medium ( $\mathrm{pH} 1.2$ ) were $0.8 \%$ to $1.2 \%$ and up to $94.9 \%$ in a buffer medium (pH 6.8) over 60 minutes [9]. Recently, LPZ enteric-coated pellets have been prepared using low substituted-HPC as a polymer for drug layering and seal coating, and Eudragit L30D-55 as the enteric-coating polymer for the dosage form. The optimized LPZ formulations' reported release from enteric-coated pellets in an acidic medium was $3.6 \%$, and the drug release in $\mathrm{pH}$ 6.8 phosphate buffer was $86 \%$ over 60 minutes. The capsules containing the LPZ pellets showed a desirable drug release in acid and buffer mediums during the stability testing period ( 3 months at $40^{\circ} \mathrm{C} / 75 \% \mathrm{RH}$ and $25^{\circ} \mathrm{C} / 60 \% \mathrm{RH}$ ) [10].

Currently, applying D-Optimal experimental design to optimize drug release from enteric-coated pellets where the coating layers are impregnated with drug for drug delivery has not been performed. The main purpose of this study was to use D-Optimal experimental design utilizing Modde 5.0 software to formulate and develop a stable, delayed release pellet formulation of LPZ with LPZ incorporated in the pellet coating, which satisfies the requirements of USP XXXV on drug dissolution in the gastrointestinal tract [11]. Then compare LPZ in vitro dissolution and in vivo bioavailability of the resultant designed LPZ formualtion to the reference product on the market (Gastevin ${ }^{\circledR}$ capsules from Slovenia).

\section{Materials and Methods}

\subsection{Chemicals}

LPZ was purchased from Jai Radhe Sales (India). Eudragit ${ }^{\circledR} 100$ was obtained as a free sample from Evonik (Germany). Lutrol F127 was provided by BASF USA (United States of America). PVA (polyvinyl alcohol) was received from Kuraray Asia Pacific Pte Ltd (Singapore). HPMC (hydroxy-propyl-methyl cellulose) E15 was obtained from Zhejiang Zhongbao (China). TEC (triethyl citrate) was purchased from Cognis (Germany). PEG (polyethylene glycol) 6000 was procured 
from Sino-Japan Chemical Co Ltd (Taiwan). Titanium dioxide (Titan Dioxide) was obtained from Cosmo (Republic of Korea). Sugar spheres were purchased from Colorcon Asia Pacific Pte Ltd (Singapore). Methanol (MeOH), acetonitrile, triethylamine and tert-butyl methyl ether were HPLC grade and purchased from Merck (Germany) and all other ingredients used were of analytical grade.

Animals and study products. The in vivo study utilized six healthy beagle dogs (between $10 \mathrm{~kg}$ and $12 \mathrm{~kg}$ in weight). The study protocol was approved by the ethics committee of medicine and pharmacy research at Military Medical University (Hanoi, Vietnam).

Test product: Thirty mg of enteric-coated LPZ loaded pellets in capsules of the optimized formulation were prepared. Reference product: Gastevin ${ }^{\circledast} 30 \mathrm{mg}$ capsules were purchased from KRKA (Slovenia) containing LPZ enteric-coated pellets, manufacture date: 02/2013, expiration date: 02/2016.

\subsection{Preparation of LPZ Enteric-Coated Pellets}

Drug loading and sub-coating. The adoption of sugar spheres as the core to load a polymer coating of drug onto to formulate the LPZ pellets, and the application of the sub-coating immediately over the drug loaded core pellets after drug loading were performed as follows. The drug coating dispersion and sub coating dispersion with LPZ and other ingredients in each formulation (Table 1) were dissolved and dispersed into a binding dispersion using $\mathrm{pH} 6.8$ phosphate buffer solution, and mixed well for at least 30 minutes. The dispersions were gently stirred during loading and coating. The listed amounts of sugar spheres (mesh size 710/850) were placed into a laboratory Diosna spray coater (Germany) with the following parameters: $1.0 \mathrm{~mm}$ nozzle-needle, atomizing pressure of $1.5-2.0$ bar, inlet air temperature of $50^{\circ} \mathrm{C}$, inlet air of $90 \%$, spray rate of $4.8-6.6$

Table 1. Formulation of drug loading and subcoating membranes of LPZ pellets.

\begin{tabular}{lcc}
\hline \multicolumn{1}{c}{ Ingredients } & Drug loading pellets & Subcoating \\
\hline LPZ (\%) & 5.70 & \\
HPMC E15 (\%) & 2.28 & 5.00 \\
PVA (\%) & 0.57 & 1.50 \\
PEG 6000 (\%) & 5.70 & \\
Dibasic sodium phosphate (\%) & 0.42 & 1.00 \\
Lutrol F127 (\%) & & 2.00 \\
Titanium dioxide (\%) & 2.28 & 2.00 \\
Talc (\%) & 100 & 100 \\
pH 6.8 phosphate buffer solution (\%) & 150 & - \\
Sugar spheres (710/850 mesh, g) & - & 20 \\
Lansoprazole loaded pellets $(\mathrm{g})^{*}$ & & \\
\hline
\end{tabular}

$\left.{ }^{*}\right)$ : Batch size for each experiment, it is not included for calculating the percentage of each ingredient. The percentage of each ingredient is compared to $100 \mathrm{~g}$ solvent. 
$\mathrm{mL} / \mathrm{min}$, and pipe diameter of $1.2 \mathrm{~mm}$ for drug loading. Sub-coating was performed with the following parameters: an atomizing pressure of $1.0 \mathrm{bar}$, an inlet air temperature of $42^{\circ} \mathrm{C}$, an inlet air of $80 \%$, a spray rate of $0.7 \mathrm{~mL} / \mathrm{min}$, and pipe diameter of $1.2 \mathrm{~mm}$. The LPZ loaded pellets were coated up to $7.5 \%$ weight gain. After finishing coating, pellets were dried for 15 minutes in a fluid bed coating system and stabilized for 24 hour.

Enteric coating. The sub-coated pellets were enteric coated using Eudragit ${ }^{\circledR}$ L100 with a batch size of $20 \mathrm{~g}$. A Wurster fluid bed coating apparatus was used (Caleva mini-fluidized bed coater, England) for 20 different D-Optimal experimental formulations designed by Modde 5.0 software (Umetrics Co., Sweden) for dissolution testing. Dispersions of required quantities of Eudragit L100, TEC, talc, and titanium dioxide in specified volumes of ethanol and purified water at a ratio of 3:1 (v/v) were prepared. The prepared dispersion was stirred during enteric coating. Coating was performed with the following parameters: an atomizing pressure of $1.2 \mathrm{bar}$, an inlet air temperature of $43^{\circ} \mathrm{C}$, an inlet air of $80 \%$, a spray rate of $0.8 \mathrm{~mL} / \mathrm{min}$, and a pipe diameter of $1.2 \mathrm{~mm}$. After coating, enteric-coated pellets were dried for 15 minutes in a fluid bed coating system and stabilized for 24 hours. The optimal formulation was selected by running the dissolution data through In Form 3.1 optimization software [12] based on the LPZ dissolution data from the experimental results and the requirements of USP XXXV.

Three batches of 3300 capsules (with each capsule containing $30 \mathrm{mg}$ LPZ, equivalent to $1000 \mathrm{~g}$ pellets per batch) of the optimal formulation were prepared to evaluate the stability of capsules containing LPZ enteric-coated pellets. The LPZ enteric-coated pellets were filled in hard gelatin capsules using a HanYang HFC45 capsule filling machine (Republic of Korea). The hard gelatin capsules were packed in aluminum blisters using an Uhlmann CP250 blister packing machine (Vietnam).

\subsection{Drug Content}

Spectrophotometric method: Drug content assays were performed in triplicate. An amount of coated pellets equivalent to $50 \mathrm{mg}$ of LPZ was weighed and put into a dry $50-\mathrm{mL}$ volumetric flask. Methanol $(\mathrm{MeOH})$ was used to dissolve the drug under sonication for 15 minutes. Then the samples were centrifuged at $5000 \mathrm{rpm}$ for 10 minutes to assure clarity of the sample for assay. Supernatant was collected and filtered through a $0.45 \mu \mathrm{m}$ Teflon membrane filter. Filtrate solutions were diluted 100 times with $\mathrm{pH} 6.8$ buffer solution. The assay performed on filtered drug solutions utilized a UV spectrophotometer (Hitachi U-1900, Japan) at $283 \mathrm{~nm}$. The amount of LPZ contained in each formulation was determined using a standard curve prepared from known standard solutions.

HPLC method: Approximately $600 \mathrm{mg}$ of enteric-coated pellets (equivalent to $60 \mathrm{mg}$ of LPZ) were placed into a dry $50-\mathrm{mL}$ volumetric flask where thirty $\mathrm{mL}$ of $\mathrm{MeOH}$ was added to the enteric-coated pellets to dissolve LPZ from the pellets 
by sonication for 15 minutes. The sample volume was made up to the mark with $\mathrm{MeOH}$, then thoroughly mixed and centrifuged at $3500 \mathrm{rpm}$ for 15 minutes. A 5 $\mathrm{ml}$ sample from the obtained supernatant filtrate solution was diluted to $50 \mathrm{~mL}$ with mobile phase, then shaken, filtered through a $0.45-\mu \mathrm{m}$ Teflon membrane filter and injected into the HPLC system. Chromatographic conditions: The steel column used was RP18 $(150 \times 4.6 \mathrm{~mm}$; $5 \mu \mathrm{m})$, with a steel pre-column (RP18, 4 $\times 3 \mathrm{~mm}$ ). Detector PDA was set at $285 \mathrm{~nm}$. The flow-rate was $1 \mathrm{~mL} / \mathrm{min}$, and the injection volume was $20 \mu \mathrm{L}$. The mobile phase was a mixture of 450:550:2.5 $(\mathrm{v} / \mathrm{v} / \mathrm{v})$ acetonitrile, water and triethylamine, $\mathrm{pH}$ adjusted to 7.0 with phosphoric acid.

\subsection{In vitro Dissolution Studies}

The release of LPZ from enteric-coated pellets in the simulated environment of the gastrointestinal tract was determined using the USP XXXV dissolution apparatus II (Erweka equipment with paddle at $37^{\circ} \mathrm{C} \pm 0.5^{\circ} \mathrm{C}$ and $75 \mathrm{rpm}$, Germany).

Acid stage ( $p H$ 1.2): LPZ release from pellets having the equivalent of $30-\mathrm{mg}$ LPZ in vessels containing $500 \mathrm{~mL}$ of $0.1 \mathrm{~N} \mathrm{HCl}$ dissolution media was determined after 1 hour. The quantity of drug in the pellets was assayed by HPLC or spectrophotometric method as follows:

HPLC method: The medium was drained without losing the pellets; HPLC method outlined above was used for determination of remaining drug in pellets.

Spectrophotometric method: Having withdrawn a $25-\mathrm{mL}$ aliquot and filtering it, the amount of drug dissolved was determined by measuring UV absorption at the wavelength of maximum absorbance at $306 \mathrm{~nm}$.

Buffer stage ( $p H 6.8$ ): $425 \mathrm{~mL}$ of buffer concentrate (4.0 L of buffer concentrate consisting of $65.4 \mathrm{~g}$ of monobasic sodium phosphate, $28.2 \mathrm{~g}$ of sodium hydroxide, $12.0 \mathrm{~g}$ of sodium dodecyl sulfate and water) was added to the remaining $475 \mathrm{~mL}$ of solution in each vessel from the acid stage. After 1 hour, the amount of drug dissolved was determined by employing HPLC or spectrophotometric method at $286 \mathrm{~nm}$.

Comparison of the two drug dissolution profiles (reference versus test formulations) was performed using the similarity factor $f_{2}$ which is calculated as follows (Equation (1)) [13]:

$$
f_{2}=50 \times \log \left\{\left[1+\left(\frac{1}{n}\right) \sum_{t=1}^{n}\left(R_{t}-T_{t}\right)^{2}\right]^{0.5} \times 100\right\}
$$

where $R_{t}$ and $T_{t}$ are the percentages of drug release at time $t$ of the reference and the test formulations, respectively; $n$ is the number of time points. If $f_{2}$ is equal to or more than 50, the two drug release profiles will be considered to be similar.

\subsection{Stability Studies}

Stability studies were carried out using 3300 capsules from batches of the optimal formulation. The optimal formulation batches were stored at various temper- 
atures: $15^{\circ} \mathrm{C}-30^{\circ} \mathrm{C} / 40-90 \% \mathrm{RH}$ (room temperature) and $40^{\circ} \mathrm{C} \pm 2{ }^{\circ} \mathrm{C} / 75 \% \pm 5 \%$ $\mathrm{RH}$ (accelerated temperature) per ICH guidelines and various physico-chemical parameters (appearance, drug content, and in vitro drug release profile) were tested periodically at $3,6,12$, and 18 months.

\subsection{In vivo Studies}

Drug administration and sample collection: This study was based on a single-dose, randomized, two-period crossover design. Six healthy beagle dogs were fed standardized meals for 3 days before inclusion into the study. The number of dogs in the study was determined from a pilot study of two doses and variance of data obtained. Each drug was taken after an overnight fast. In the morning of phase I, three randomly chosen dogs were given a single dose of reference product and three other dogs were given a single dose of test product with $100 \mathrm{~mL}$ of water. No food was allowed until 1 hour after collection of the final blood sample. Water intake was allowed after 4 hours dose administration. Phase II was conducted 72 hours after finishing the blood sample collection of phase I. The process of phase II was carried out inversely with respect to the animals and study products. Approximately $3 \mathrm{ml}$ blood samples were drawn into heparinized tubes through sterile syringes from the jugular vein before LPZ administration $(0 \mathrm{~h})$ and at $0.5,1.0,1.5,2.0,2.25,2.5,2.75,3.0,3.5,4.0,6.0,8.0,10,12$ and $24 \mathrm{~h}$ after dosing for LPZ chromatographic assay. The blood samples were centrifuged at $5000 \mathrm{rpm} / \mathrm{min}$ for 7 minutes. Plasma samples were separated and kept frozen at $-45^{\circ} \mathrm{C}$ until assay.

Extraction of LPZ from plasma: $50-\mu \mathrm{L}$ internal standard solution of pantoprazole $(40 \mu \mathrm{g} / \mathrm{mL})$ and 2 -mL tert-butyl methyl ether were added to $500 \mu \mathrm{L}$ of each plasma sample. The solution was extracted by vortex mixing for 3 minutes, followed by centrifugation at $4500 \mathrm{rpm} / \mathrm{min}$ for 10 minutes at $20^{\circ} \mathrm{C}$. A $1-\mathrm{mL}$ aliquot of the supernatant obtained was transferred to a glass tube and evaporated until dry at $30^{\circ} \mathrm{C}$. The residue was dissolved in a solution containing $80-\mu \mathrm{L}$ acetonitrile and $120-\mu \mathrm{L} 0.01 \mathrm{M}$ potassium dihydro phosphate buffer solution ( $\mathrm{pH}$ adjusted to 8.0 with triethylamine) and mixed for 2 minutes. A $50-\mu \mathrm{L}$ aliquot was subsequently injected into the HPLC system.

Chromatographic conditions. HPLC separation was carried out using a RP18 steel column $(150 \times 4.6 \mathrm{~mm} ; 5 \mu \mathrm{m})$ preceded by a steel guard column $(\mathrm{RP} 18,4 \times$ $3 \mathrm{~mm}$ ). The detector UV used was set at $285 \mathrm{~nm}$. The flow-rate was $1 \mathrm{~mL} / \mathrm{min}$, injection volume was $50 \mu \mathrm{L}$. The mobile phase was a mixture of 65:35 (v/v) 0.01 $\mathrm{M}$ potassium dihydrophosphate buffer solution ( $\mathrm{pH}$ adjusted to 8.0 with triethylamine) and acetonitrile.

Pharmacokinetic and statistical analysis: The pharmacokinetic parameters of LPZ in beagle dogs given capsules containing enteric-coated pellets (test product) and the reference product were calculated using the noncompartmental pharmacokinetic analysis method with the aid of WinNonlin 5.2 software (Certara Inc., USA). $\mathrm{C}_{\max }$ and $\mathrm{T}_{\max }$ were obtained directly from the observed concentration-time data. The area under the curve to the last measurable concentration 
$\left(\mathrm{AUC}_{0-t}\right)$ was calculated by the linear trapezoidal rule. The area under the curve extrapolated to infinity $\left(\mathrm{AUC}_{0-\infty}\right)$ was calculated by the following formula: $\mathrm{AUC}_{0-\infty}=\mathrm{AUC}_{0-t}+C_{t} / K_{e b}$ where $\mathrm{C}_{\mathrm{t}}$ is the last measurable concentration and $K_{e l}$ is the elimination rate constant. All values are expressed as the mean values \pm standard deviation. For the purpose of bioequivalence analysis, $\mathrm{C}_{\max }, \mathrm{AUC}_{0-t}$ and $\mathrm{AUC}_{0-\infty}$ were considered as primary variables. Bioequivalence was assessed using analysis of variance for crossover design and calculating standard $90 \%$ confidence intervals of the ratio test/reference. The products were considered bioequivalent if the difference between two compared parameters was found statistically insignificant $(\mathrm{P} \geq 0.05)$ and $90 \%$ confidence intervals for these parameters fell within $80 \%-120 \%$.

\section{Results}

\subsection{Formulation of LPZ Enteric-Coated Pellets}

Effects of the independent variables on the response variables. LPZ entericcoated pellets were prepared by using Eudragit ${ }^{\oplus} \mathrm{L} 100$ as the enteric coating polymer. The independent variables and the range of levels incorporated in to the test formulations are shown in Table 2. The amount of titanium dioxide was fixed at $20 \%$ of enteric polymer. The volume of ethanol/purified water solution used was $100 \mathrm{~mL}$ per formulation. The amount of Eudragit ${ }^{\circ} \mathrm{L} 100$ was fixed at 7.5 g per $100 \mathrm{~mL}$ coating solution. D-optimal experimental design by Modde 5.0 software was used to create 20 different experimental formulations of LPZ enteric-coated pellets (Table 3). The percentage of TEC (X1), the percentage of talc (X2) and the weight gain of enteric coating membrane (X3) were three independent variables (the percentage of TEC or talc was calculated as the percent of each ingredient per enteric polymer). Dependent variables were the percentages of drug released in acid dissolution media (Y1) and buffer dissolution media (Y2).

Table 3 also shows the results of dissolution in acid and buffer media ( $\mathrm{pH} 1.2$ and $\mathrm{pH} 6.8$, respectively). From the data obtained, the response surface analysis shows the influence of the input variables on the output variables through the aid of In Form 3.1 optimization software. Figure 1 and Figure 2 show the correlation between input variables (percentage of TEC, percentage of talc and weight gain of enteric-coating membrane) and percentage of $\mathrm{LPZ}$ released in $\mathrm{pH}$ 1.2 media and $\mathrm{pH} 6.8$ media, respectively.

Table 2. Independent variables and percentage range of TEC, talc and weight gain of pellets examined.

\begin{tabular}{ccc}
\hline Factors & Lower Percentage $(-)$ & Upper Percentage $(+)$ \\
\hline $\mathrm{X} 1$ & 20 & 30 \\
$\mathrm{X} 2$ & 30 & 50 \\
$\mathrm{X} 3$ & 25 & 35 \\
\hline
\end{tabular}

$\mathrm{X} 1$ percentage of TEC; $\mathrm{X} 2$ percentage of talc; $\mathrm{X} 3$ weight gain of enteric-coated membrane. 
Table 3. Formulation and dissolution data of LPZ enteric-coated pellets in $\mathrm{pH} 1.2$ (Y1) and pH 6.8 (Y2).

\begin{tabular}{|c|c|c|c|c|c|}
\hline No & X3 (\%) & $\mathrm{X} 1(\%)$ & X2 (\%) & Y1 (\%) & Y2 (\%) \\
\hline N1 & 25.34 & 20.00 & 30.00 & 9.70 & 81.14 \\
\hline N2 & 34.47 & 20.00 & 30.00 & 5.32 & 85.60 \\
\hline N3 & 35.09 & 20.00 & 50.00 & 3.08 & 87.31 \\
\hline N4 & 26.97 & 20.00 & 36.67 & 4.55 & 87.96 \\
\hline N5 & 32.14 & 20.00 & 50.00 & 1.52 & 92.25 \\
\hline N6 & 29.48 & 20.00 & 50.00 & 7.92 & 84.26 \\
\hline N7 & 25.88 & 20.00 & 43.33 & 9.17 & 78.95 \\
\hline N8 & 24.79 & 23.33 & 50.00 & 9.78 & 77.49 \\
\hline N9 & 34.70 & 25.00 & 40.00 & 8.16 & 86.08 \\
\hline N10 & 33.44 & 20.00 & 40.00 & 6.38 & 85.96 \\
\hline N11 & 33.44 & 25.00 & 30.00 & 11.91 & 72.64 \\
\hline $\mathrm{N} 12$ & 29.94 & 25.00 & 40.00 & 8.49 & 85.20 \\
\hline N13 & 30.61 & 25.00 & 40.00 & 6.69 & 87.81 \\
\hline N14 & 29.40 & 25.00 & 40.00 & 6.47 & 82.69 \\
\hline N15 & 30.54 & 25.00 & 40.00 & 8.30 & 83.14 \\
\hline N16 & 26.04 & 30.00 & 50.00 & 8.49 & 73.79 \\
\hline N17 & 24.23 & 30.00 & 30.00 & 14.54 & 70.50 \\
\hline N18 & 34.15 & 30.00 & 30.00 & 6.58 & 90.37 \\
\hline N19 & 25.56 & 26.67 & 50.00 & 8.48 & 84.10 \\
\hline $\mathrm{N} 20$ & 34.89 & 30.00 & 50.00 & 4.69 & 83.08 \\
\hline
\end{tabular}

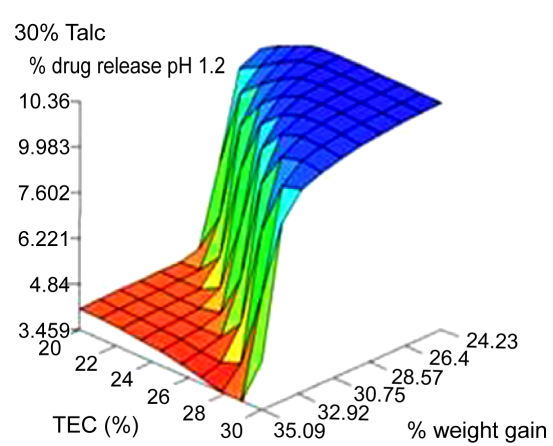

(a)

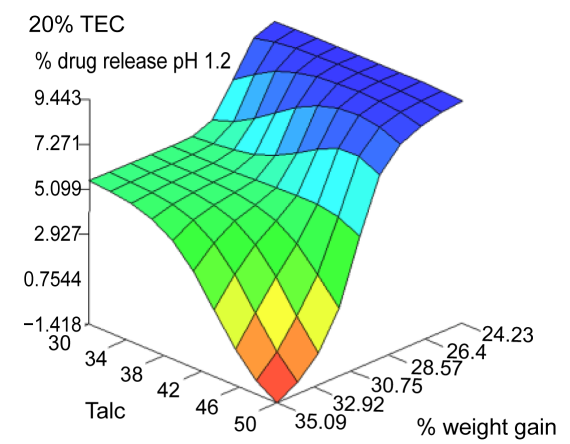

(b)

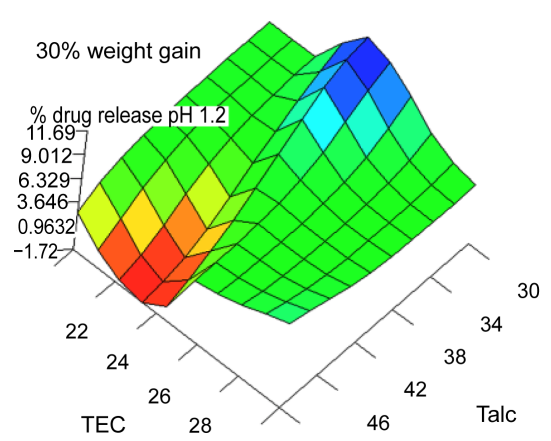

(c)

Figure 1. Response surface plots of percentage drug release in $\mathrm{pH} 1.2$ medium versus input variables, (1a); TEC\% added and \%weight gain, (1b); Talc added and \% weight gain, and (1c); TEC added and Talc added.

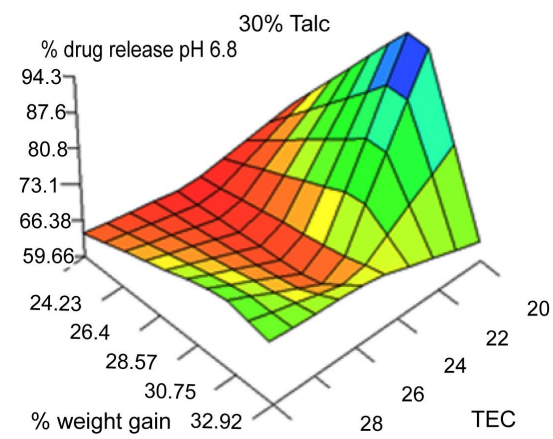

(a)

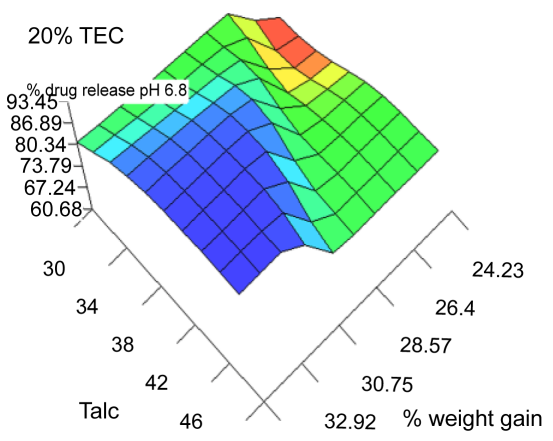

(b)

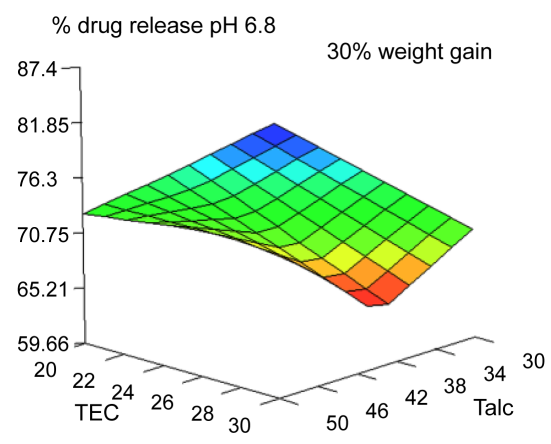

(c)

Figure 2. Response surface plots of percentage drug release in $\mathrm{pH} 6.8$ medium versus input variables, (2a); TEC\% added and \%weight gain, (2b); Talc added and \% weight gain, and (2c); TEC added and Talc added. 
Figure 1 shows that at 30\% talc (Figure 1(a)), the acid resistance of LPZ enteric-coated pellets is in direct proportion to the weight gain of the enteric-coating membrane. In the case of the weight gain of enteric-coating membrane below 26\%, the drug released in $\mathrm{pH} 1.2$ was approximately $10 \%$. An increase in percentage of LPZ release is directly proportional to the percentage of TEC at the same weight gain of enteric-coating membrane. The highest acid resistance of LPZ enteric-coated pellets was recorded when the percentage of TEC was below $24 \%$ and the weight gain of enteric-coating membrane was between $30 \%$ - 35\%. At 20\% TEC (Figure 1(b)), the percent LPZ dissolved in acid dissolution media from enteric-coated pellets is inversely proportional to the percentage of talc. At this point, increasing a weight gain of enteric-coating membrane induces a decrease in drug released in $\mathrm{pH} 1.2$ dissolution media. Figure 1(c) also shows that the lowest LPZ released from the pellets was at $\mathrm{pH} 1.2$ and the percentage of talc above $40 \%$ while the percentage of TEC was between $22 \%-24 \%$.

Figure 2 shows the correlation between input variables and percentage of LPZ release in $\mathrm{pH} 6.8$ dissolution media and drug remaining after finishing the acid resistance test. At 30\% talc (Figure 2(a)), the highest dissolution of LPZ in buffer solution from enteric-coated pellets was recorded when the percentage of TEC was $22 \%$ and the weight gain of enteric-coating membrane was between 29 - $31 \%$. Using TEC at high ratios (above $26 \%$ ) possibly caused the decrease in LPZ released at $\mathrm{pH} 6.8$ because the drug released at $\mathrm{pH} 1.2$ reached a high level. Similarly, at 20\% TEC (Figure 2(b)), the weight gain of enteric-coating membrane and the percentage of talc was below $25 \%$ and $35 \%$ respectively. The decrease of LPZ released in $\mathrm{pH} 6.8$ dissolution media correlated to the increase of drug released in the $\mathrm{pH} 1.2$ dissolution media. The highest dissolution of LPZ from enteric-coated pellets in buffer solution was recorded when the percentage of talc was above $42 \%$ and the weight gain of enteric-coating membrane was above $30 \%$. Figure 2 (c) shows that the drug released at $\mathrm{pH} 6.8$ rose when the amount of talc and TEC increased concurrently with a $30 \%$ weight gain of enteric-coating membrane.

Optimization of the formulation of LPZ enteric-coated pellets. Based on the experimental dissolution data, the range of optimal conditions for dependent variables are as follows: the percentages of drug released in acid $\mathrm{pH} 1.2$ medium $(0 \% \leq \mathrm{Y} 1 \leq 10 \%)$, and the percentages of drug released in buffer $\mathrm{pH} 6.8$ medium $(80 \% \leq \mathrm{Y} 2 \leq 100 \%)$ were identified. Running In Form 3.1 optimization software program, the optimal formulation of enteric coating was extrapolated and shown in Table 4 with the predicted results of their dissolution in acid pH 1.2 medium and buffer $\mathrm{pH} 6.8$ dissolution medium being $4.07 \%$ and $90.87 \%$, respectively.

The optimal formulation of enteric coating was prepared at the batch size of $150 \mathrm{~g}(\mathrm{n}=3)$ using Diosna spray coater (Germany). The enteric membrane was coated on the subcoating core pellets containing LPZ. The physico-chemical properties of enteric-coated pellets for optimal formulation were evaluated and shown in Table 5. The obtained results show that the optimal enteric-coated pellets had good flowability (flow rate $=11.8 \mathrm{~g} / \mathrm{s}$ ), and suitable particle size dis- 
tribution for filling in hard gelatin capsules $(0.85-1.2 \mathrm{~mm})$. The assayed content of drug in the pellets was approximately $8 \%$. The moisture, friability, and bulk density of enteric-coated pellets were in an acceptable range.

The dissolution profiles of optimal formulation pellets were the same as that of Gastevin ${ }^{\circledR} 30 \mathrm{mg}$ with $f_{2}$ equal to 56.62 (Figure 3). Both the optimized test formulation and Gastevin ${ }^{\circledR} 30 \mathrm{mg}$ completely released LPZ over 60 minutes in pH 6.8 buffer solution (after testing its acid-resistance capability in $\mathrm{pH} 1.2$ acidic solution for 60 minutes). The gastric resistance of both formulations was below $5 \%$ (3.35\% for the test formulation, $3.61 \%$ for the reference). The dissolution results of the drug release profile from prepared LPZ enteric-coated pellets satisfied the requirements of USP XXXV on drug dissolution in in vitro gastrointestinal tract.

Table 4. The predicted results of optimal formulation of enteric coating.

\begin{tabular}{cc}
\hline Ingredients & Percentage (\%) \\
\hline TEC $^{*}$ & 20.00 \\
Talc $^{*}$ & 46.14 \\
Titan dioxide* & 20.00 \\
Weight gain of enteric-coating membrane & 35.09 \\
\hline
\end{tabular}

$\left.{ }^{*}\right)$ : percent per enteric polymer, using the mixture of ethanol/purified water $(3: 1)$ as solvent for coating; Titanium Oxide was held constant to amount of the Enteric-coated polymer used in the study.

Table 5. Physico-chemical properties of LPZ enteric-coated pellets for optimal formulation $(\mathrm{n}=3)$.

\begin{tabular}{ccccccc}
\hline \multicolumn{7}{c}{ Pellet characteristics } \\
\hline Shape & $\begin{array}{c}\text { Particle size } \\
\text { distribution } \\
(\mathrm{mm})\end{array}$ & $\begin{array}{c}\text { Moisture } \\
(\%)\end{array}$ & $\begin{array}{c}\text { Bulk density } \\
(\mathrm{g} / \mathrm{mL})\end{array}$ & $\begin{array}{c}\text { Flow rate } \\
(\mathrm{g} / \mathrm{s})\end{array}$ & $\begin{array}{c}\text { Friability } \\
(\%)\end{array}$ & $\begin{array}{c}\text { Drug } \\
\text { content } \\
(\%)\end{array}$ \\
\hline $\begin{array}{c}\text { Spherical } \\
\text { and smooth } \\
\text { uniformity }\end{array}$ & $0.85-1.2$ & $3.58 \pm 0.78$ & $0.81 \pm 0.04$ & $11.8 \pm 0.42$ & 0.05 & $8.26 \pm 0.35$ \\
\hline
\end{tabular}

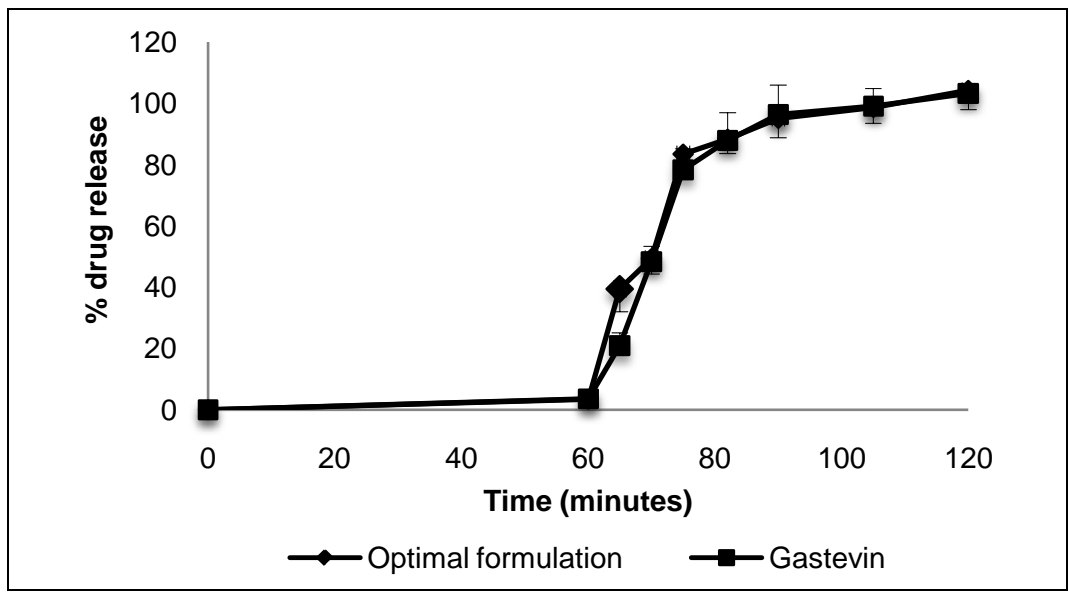

Figure 3. Dissolution profiles of Gastevin ${ }^{\circ} 30 \mathrm{mg}$ and $30 \mathrm{mg}$ capsules containing optimal formulation of LPZ enteric-coated pellets $(n=12)$. 
The scanning electron microscopy (SEM) of the optimal formulation shows that prepared enteric-coated pellets have a good coating with three individual layers including a drug loading, a subcoating, and an enteric coating (Figure 4). The thickness of each layer was estimated as $50-80 \mu \mathrm{m}$ for drug loading and enteric coating, and $10-20 \mu \mathrm{m}$ for subcoating. The layers are effective in controlling the release of LPZ in acidic and buffer dissolution media.

Stability studies: Triple batches of 3300 capsules containing the optimal formulation of LPZ enteric-coated pellets were prepared by the same method. The results of dissolution and drug content from the stability study in accelerated and room conditions are summarized in Table 6. The results reveal that the prepared capsules containing LPZ enteric-coated pellets were stable for 18 months at room conditions and 6 months in accelerated conditions.

\subsection{In Vivo Study of Capsules Containing LPZ Enteric-Coated Pellets}

The mean concentration-time profiles of the LPZ 30-mg test and reference capsules are depicted in Figure 5. The pharmacokinetic parameters for both formulations are shown in Table 7. For bioequivalence evaluation, $\mathrm{C}_{\max }, \mathrm{AUC}_{0-t}$ and $\mathrm{AUC}_{0-\infty}$ were considered as primary variables for statistical analysis. Various statistical models were applied to the pharmacokinetic parameters per FDA guidelines [14]. The statistical significant differences in the pharmacokinetic parameters between two products were analyzed by ANOVA (analysis of variance) using WinNonlin 5.2 software. The results of the statistical analysis for $\mathrm{C}_{\max }$, $\mathrm{AUC}_{0-\infty}$ and $\mathrm{AUC}_{0-\infty}$ are shown in Table 8. In addition, $\mathrm{T}_{\max }$ was analyzed by non-parametric statistical hypothesis test (Wilcoxon signed-rank test with the results in Table 9).

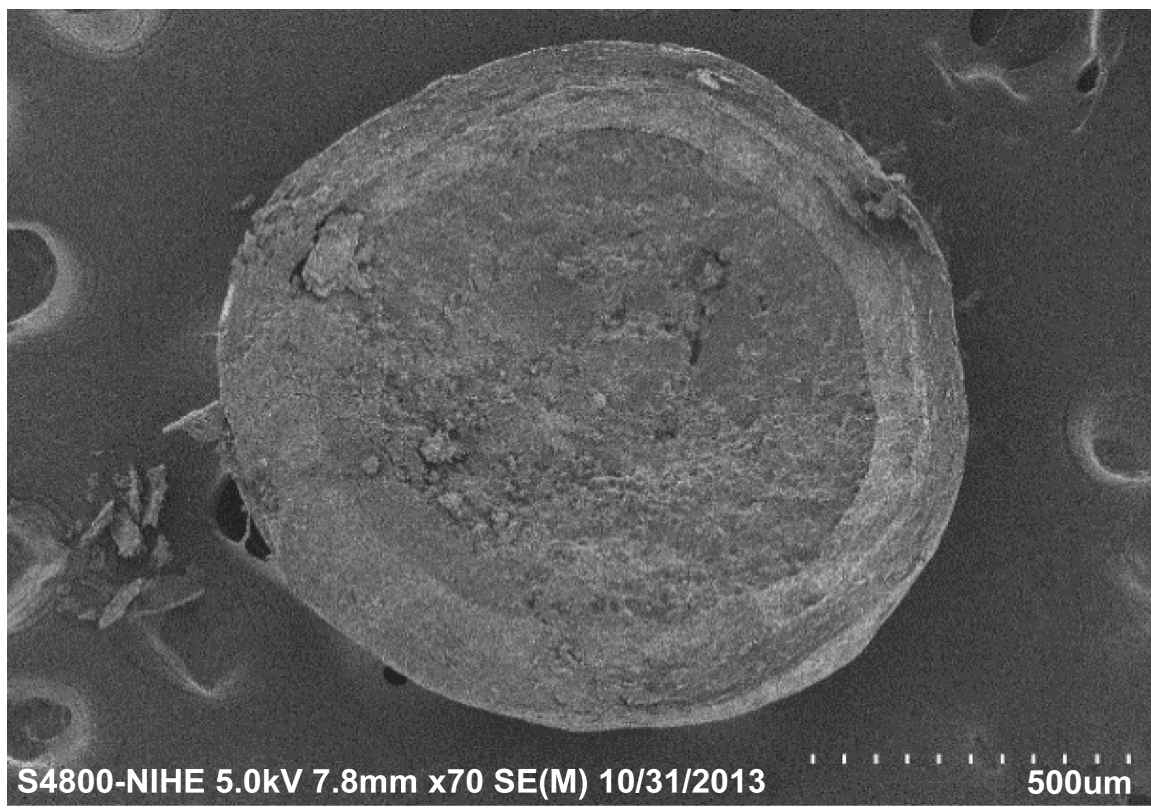

Figure 4. SEM of optimal formulation of LPZ enteric-coated pellets showing three coating layers around the sugar bead core. 
Table 6. Contents and dissolution of LPZ from optimal formulation in different storage conditions.

\begin{tabular}{|c|c|c|c|c|c|}
\hline \multirow{2}{*}{$\begin{array}{c}\text { Time } \\
\text { (months) }\end{array}$} & \multirow{2}{*}{ Sample } & & \multirow{2}{*}{$\begin{array}{l}\text { Drug content }(\%) \\
\qquad(\mathrm{n}=3)\end{array}$} & \multicolumn{2}{|c|}{ Percent of LPZ release $(n=6)$} \\
\hline & & & & Y1 & $\mathrm{Y} 2$ \\
\hline \multirow{3}{*}{$t=0$} & B1 & & $102.93 \pm 1.02$ & $3.09 \pm 0.48$ & $96.94 \pm 1.96$ \\
\hline & $\mathrm{B} 2$ & & $102.45 \pm 1.27$ & $3.49 \pm 0.20$ & $98.92 \pm 1.70$ \\
\hline & B3 & & $101.98 \pm 2.11$ & $3.39 \pm 0.07$ & $97.26 \pm 1.63$ \\
\hline \multirow{6}{*}{$t=3$} & B1 & rc & $101.84 \pm 0.61$ & $2.91 \pm 0.46$ & $96.41 \pm 1.85$ \\
\hline & B1 & ac & $101.29 \pm 1.32$ & $3.10 \pm 0.30$ & $98.68 \pm 1.60$ \\
\hline & B2 & $\mathrm{rc}$ & $100.09 \pm 0.29$ & $3.08 \pm 0.24$ & $98.93 \pm 1.62$ \\
\hline & B2 & ac & $100.99 \pm 1.76$ & $3.64 \pm 0.40$ & $99.59 \pm 1.27$ \\
\hline & B3 & $\mathrm{rc}$ & $101.60 \pm 2.63$ & $3.07 \pm 0.22$ & $99.66 \pm 2.18$ \\
\hline & B3 & ac & $101.69 \pm 1.03$ & $3.57 \pm 0.39$ & $99.42 \pm 1.35$ \\
\hline \multirow{6}{*}{$t=6$} & B1 & $\mathrm{rc}$ & $101.50 \pm 1.09$ & $2.98 \pm 0.38$ & $96.00 \pm 1.26$ \\
\hline & B1 & ac & $99.52 \pm 1.18$ & $3.67 \pm 0.28$ & $98.58 \pm 1.19$ \\
\hline & B2 & $\mathrm{rc}$ & $99.93 \pm 0.52$ & $3.45 \pm 0.23$ & $98.08 \pm 1.26$ \\
\hline & B2 & ac & $101.96 \pm 0.10$ & $3.92 \pm 0.24$ & $98.84 \pm 1.88$ \\
\hline & B3 & $\mathrm{rc}$ & $101.94 \pm 2.53$ & $3.43 \pm 0.12$ & $98.54 \pm 1.92$ \\
\hline & B3 & ac & $99.48 \pm 1.09$ & $3.75 \pm 0.43$ & $98.62 \pm 0.78$ \\
\hline \multirow{3}{*}{$\mathrm{t}=12$} & B1 & $\mathrm{rc}$ & $102.21 \pm 0.81$ & $3.31 \pm 0.27$ & $98.85 \pm 1.15$ \\
\hline & B2 & $\mathrm{rc}$ & $101.93 \pm 1.03$ & $3.38 \pm 0.34$ & $99.33 \pm 1.14$ \\
\hline & B3 & $\mathrm{rc}$ & $99.77 \pm 1.82$ & $3.35 \pm 0.28$ & $98.92 \pm 0.89$ \\
\hline \multirow{3}{*}{$\mathrm{t}=18$} & B1 & $\mathrm{rc}$ & $100.32 \pm 0.93$ & $4.08 \pm 0.21$ & $94.72 \pm 1.32$ \\
\hline & B2 & $\mathrm{rc}$ & $100.75 \pm 1.25$ & $4.96 \pm 0.38$ & $93.63 \pm 1.59$ \\
\hline & B3 & $\mathrm{rc}$ & $99.25 \pm 1.08$ & $4.18 \pm 0.58$ & $95.85 \pm 1.36$ \\
\hline
\end{tabular}

$\mathrm{rc}=$ room condition, $\mathrm{ac}=$ accelerated condition $; \mathrm{B} 1=$ batch $1, \mathrm{~B} 2=$ batch $2, \mathrm{~B} 3=$ batch 3.

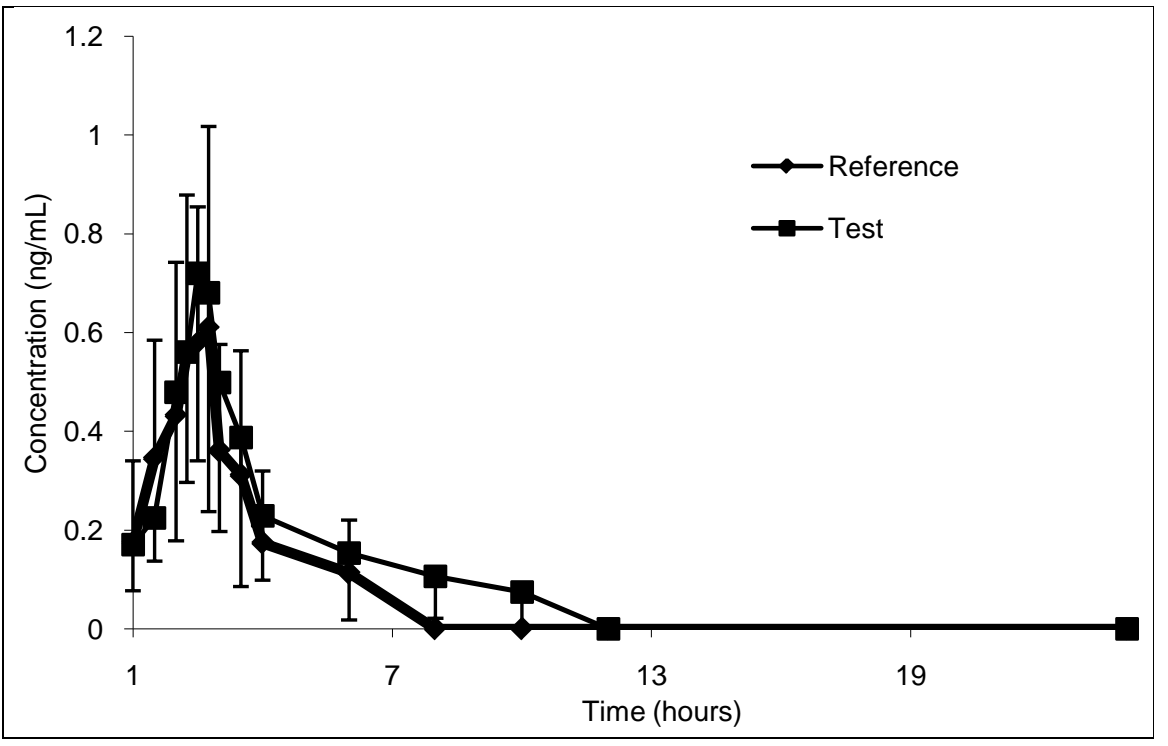

Figure 5. Plasma concentration-time profile of LPZ 30-mg capsules (Gastevin and optimal formulation). 
Table 7. Pharmacokinetic parameters of the two products of LPZ 30-mg capsules.

\begin{tabular}{ccccccccc}
\hline \multirow{2}{*}{ Animal no. } & \multicolumn{2}{c}{$\mathrm{C}_{\max }(\mu \mathrm{g} / \mathrm{mL})$} & \multicolumn{2}{c}{$\mathrm{T}_{\max }(\mathrm{h})$} & \multicolumn{2}{c}{$\mathrm{AUC}_{0-t}(\mathrm{ng} / \mathrm{mL} / \mathrm{h})$} & \multicolumn{2}{c}{$\mathrm{AUC}_{0-\infty}(\mathrm{ng} / \mathrm{mL} / \mathrm{h})$} \\
\cline { 2 - 9 } & $\mathrm{R}$ & $\mathrm{T}$ & $\mathrm{R}$ & $\mathrm{T}$ & $\mathrm{R}$ & $\mathrm{T}$ & $\mathrm{R}$ & $\mathrm{T}$ \\
\hline 1 & 1.0515 & 1.4823 & 2.25 & 2.75 & 3194.9 & 3627.4 & 3330.4 & 3660.4 \\
2 & 0.7851 & 1.0238 & 2.25 & 2.00 & 1866.0 & 1518.1 & 2021.4 & 1543.7 \\
3 & 0.7111 & 0.4310 & 2.50 & 2.00 & 1998.0 & 784.2 & 2120.3 & 889.2 \\
4 & 0.4320 & 0.8134 & 2.00 & 2.75 & 450.8 & 2040.1 & 474.6 & 2158.4 \\
5 & 1.2963 & 1.1512 & 2.75 & 2.50 & 1651.5 & 2964.1 & 1939.5 & 3457.5 \\
6 & 0.5251 & 0.6540 & 2.25 & 2.25 & 1031.9 & 2537.8 & 1069.6 & 3359.4 \\
Mean & 0.8002 & 0.9260 & 2.33 & 2.37 & 1698.8 & 2245.2 & 1825.9 & 2511.4 \\
$\mathrm{SD}$ & 0.3252 & 0.3746 & 0.25 & 0.34 & 933.8 & 1021.5 & 979.7 & 1151.2 \\
\hline
\end{tabular}

$\mathrm{R}=$ reference product; $\mathrm{T}=$ test product.

Table 8. Statistical analysis of the pharmacokinetic data of LPZ 30-mg capsules.

\begin{tabular}{ccccccc}
\hline Dependent & Hypothesis & DF & SS & MS & F_stat & P_value \\
\hline $\operatorname{Ln}\left(\mathrm{C}_{\max }\right)$ & Sequence & 1 & 0.0478 & 0.0478 & 0.14 & 0.7284 \\
$\operatorname{Ln}\left(\mathrm{C}_{\max }\right)$ & Sequence Subject & 4 & 1.3774 & 0.3443 & 3.83 & 0.1107 \\
$\operatorname{Ln}\left(\mathrm{C}_{\max }\right)$ & Formulation & 1 & 0.0590 & 0.0590 & 0.66 & 0.4630 \\
$\operatorname{Ln}\left(\mathrm{C}_{\max }\right)$ & Period & 1 & 0.0326 & 0.0326 & 0.36 & 0.5792 \\
$\operatorname{Ln}\left(\mathrm{AUC}_{0-t}\right)$ & Sequence & 1 & 0.1809 & 0.1809 & 0.41 & 0.5574 \\
$\operatorname{Ln}\left(\mathrm{AUC}_{0-t}\right)$ & Sequence Subject & 4 & 1.7703 & 0.4426 & 3.43 & 0.1299 \\
$\operatorname{Ln}\left(\mathrm{AUC}_{0-t}\right)$ & Formulation & 1 & 0.3267 & 0.3267 & 2.53 & 0.1868 \\
$\operatorname{Ln}\left(\mathrm{AUC}_{0-t}\right)$ & Period & 1 & 1.3394 & 1.3394 & 10.38 & 0.0322 \\
$\operatorname{Ln}\left(\mathrm{AUC}_{0-\infty}\right)$ & Sequence & 1 & 0.0948 & 0.0948 & 0.21 & 0.6740 \\
$\operatorname{Ln}\left(\mathrm{AUC}_{0-\infty}\right)$ & Sequence Subject & 4 & 1.8481 & 0.4620 & 4.03 & 0.1030 \\
$\operatorname{Ln}\left(\mathrm{AUC}_{0-\infty}\right)$ & Formulation & 1 & 0.4008 & 0.4008 & 3.49 & 0.1350 \\
$\operatorname{Ln}\left(\mathrm{AUC}_{0-\infty}\right)$ & Period & 1 & 1.5275 & 1.5275 & 13.31 & 0.0218 \\
\hline
\end{tabular}

The pharmacokinetic data were transformed into natural logarithm (Ln); DF = degrees of freedom; SS = sum of squares; MS = mean square.

Table 9. Wilcoxon signed-rank test for $\mathrm{T}_{\max }$.

\begin{tabular}{|c|c|c|c|c|}
\hline \multirow{2}{*}{ Animal no. } & \multicolumn{2}{|c|}{$\mathrm{T}_{\max }(\mathrm{h})$} & \multirow{2}{*}{ Difference } & \multirow{2}{*}{ Ranked difference } \\
\hline & $\mathrm{R}$ & $\mathrm{T}$ & & \\
\hline 1 & 2.25 & 2.75 & -0.5 & $3.5(3-4)$ \\
\hline 2 & 2.25 & 2.00 & +0.25 & $1.5(1-2)$ \\
\hline 3 & 2.50 & 2.00 & +0.5 & $3.5(3-4)$ \\
\hline 4 & 2.00 & 2.75 & -0.75 & 5 \\
\hline 5 & 2.75 & 2.50 & +0.25 & $1.5(1-2)$ \\
\hline 6 & 2.25 & 2.25 & 0 & \\
\hline
\end{tabular}


$C_{\max }$ and $T_{\max }$ : The mean $\mathrm{C}_{\max }$ was $0.800 \pm 0.325$ and $0.926 \pm 0.374 \mu \mathrm{g} / \mathrm{mL}$ for reference and test products, respectively. $90 \%$ confidence interval ranges between the reference and test products fell within $79.56 \%-166.40 \%$. At the 0.95 significance level, ANOVA did not show any significant differences between the two products on all effects. For example, the effects of sequence on $\mathrm{C}_{\max }$, the observed $P$ value was 0.728 while the $P$ value was 0.110 for the influence of study subjects on $\mathrm{C}_{\max }$. In terms of treatment, no significant differences were seen, (the observed $P$ value was 0.463 ) and the period effects with the observed $P$ value were 0.579 .

For $\mathrm{T}_{\max }$, the sum of the ranks of the scores with positive and negative values were 6.5 and 8.5 , respectively. Therefore, the smaller sum was 6.5 and the number of differences was 5 . Using the table of Wilcoxon signed-rank, no significant difference was recorded at the 0.95 significance level $(P>0.05)$. With the value of $\mathrm{T}_{\max }$ obtained and dissolution data in acidic medium, the LPZ 30-mg test capsules showed delayed release in gastrointestinal tract on 6 beagle dogs in this in vivo study.

$A U C_{0-t}$ and $A U C_{0-\infty}$ : The mean $\mathrm{AUC}_{0-t}$ were $1698.8 \pm 933.8$ and $2245.2 \pm$ $1021.5 \mathrm{ng} / \mathrm{mL} / \mathrm{h}$ for reference and test products, respectively. $90 \%$ confidence interval range between the reference and test products fell within 89.38 $216.46 \%$. At the 0.95 significance level, ANOVA did not show any significant differences between the two products on the effects of sequence on $\mathrm{AUC}_{0-t}(P$ value $=0.557)$, the effects of subject $(P$ value $=0.129)$, and the effects of treatment $(P$ value $=0.186)$. However, there was a statistically significant difference between the test and reference products on period effects $(P$ value $<0.05)$. The same results were obtained for $\mathrm{AUC}_{0-\infty}$.

To evaluate the bioavailability of prepared LPZ 30-mg enteric capsules, the in vivo study was conducted based on a single-dose, randomized, two-period crossover design per FDA guidelines. The mean concentration-time profiles of the LPZ 30-mg test and reference capsules showed their delayed-release characteristics in gastrointestinal tract on experimental dogs. With the obtained values of $\mathrm{C}_{\max }, \mathrm{AUC}_{0-\infty}$ and $\mathrm{AUC}_{0-\infty}$ by utilizing the noncompartmental method, the lack of bioequivalence outcome was given between the test and reference products whereas $\mathrm{T}_{\max }$ of both formulations was equivalent. Although the in vitro dissolution profiles of the two products were similar with $f_{2}$ value at 56.62, but the obtained in vivo results were not equivalent which might be caused by the low $f_{2}$ and the small number of subjects in the in vivo study. The pharmacokinetic parameters of the two compared capsules were appropriate to the results of previous research [15] [16]. However, due to being limited to using healthy beagle dogs as experimental subjects for the in vivo study and without the option for human subjects might have affected the in vivo bioequivalence assessment.

\section{Discussion and Summary}

LPZ is characterized by low solubility and low stability. In this study, the drug layering method was selected to prepare the core pellets containing LPZ. The 
results also show that the solubility and stability of LPZ increased considerably by layering and including alkaline salts, similarly seen in previous research [10] [17] [18]. These studies loaded the drug in the core pellet and reported that 8 percent loading gave the best release profile from the composition of the drug loaded pellets. In the optimized formulation of this study HPMC E15 and PVA were used as a binder, and dibasic sodium phosphate was used as stabilizer for protecting the drug against degradation. Lutrol F127 is a block copolymer referred to as Poloxamer 407, and was used as a solubilizer for LPZ. Sugar spheres were used as an inert pellet core for loading the drug and other ingredients onto. Due to the strong degradation of LPZ in highly acidic environments, the enteric coating was applied on the LPZ pellets after drug loading and subcoating. Eudragit ${ }^{\circ} \mathrm{L} 100$ was used as enteric coating polymer in the composition of enteric-coated pellets. However, because of Eudragit ${ }^{\circ}$ L100's acidity, it is necessary to protect the drug inside the LPZ core pellets from the acidic impact of Eudragit ${ }^{\circ}$ L100. For this reason, the subcoating was applied onto the LPZ core pellets. PVA was selected as a water-soluble polymer and PEG 6000 also was used as plasticizer. In addition, Lutrol F127 was continually used in formulation of the subcoating because of its anti-humidity effects [19]. All ingredients of layering and subcoating were compatible with LPZ.

As is shown in many earlier studies, some enteric polymers and aqueous dispersions such as HPMCP, HPMCAS, Eudragit ${ }^{\oplus}$ L30D-55, Acrylcoat ${ }^{\oplus}$ L30D have been used for coating derivatives of benzimidazoles [8] [18] [20] [21]. LPZ release from enteric coated pellets were found to be adequately improved by adjusting the amounts of TEC, talc and weight gain of pellets by the coating material [20] [21]. Therefore, these formulation parameters were used in the DOptimal experimental design at the independent variables to optimize the formulation. Release of LPZ from the optimized pellets occurred at the optimal loading of LPZ which was 8.45 which was similar to previous studies [20] [21]. A simple layering coating was applied to nonpareil pellets with LPZ provided good release of LPZ, in this study an optimized coating layer with LPZ was applied around sugar spheres to provide optimal drug release from the final optimized formulation [20]. In this study, Eudragit L100 was dissolved into a mixture of ethanol/purified water (3:1) to form the stable enteric-coating solution. To formulate the optimal LPZ enteric-coated pellets suitable for the requirements of USP XXXV on drug dissolution in gastrointestinal tract (The USP 2013) [11], 20 different experimental preparations were designed with a polynomial statistical model by Modde 5.0 software with D-optimal mixture. Three independent variables were selected as the percentage of TEC (X1), the percentage of talc (X2) and the weight gain of enteric-coating membrane (X3). By the support of a mathematical model, the In Form 3.1 optimization software brought out the optimal percentage of three input variables and predicted the values of output variables ( $\mathrm{Y} 1$ as the percentage of drug released in acid $\mathrm{pH} 1.2$ medium, and $\mathrm{Y} 2$ as the percentage of drug released in buffer $\mathrm{pH} 6.8$ medium). The experimental results showed that the enteric coating is helpful to decrease drug release in acid 
medium and increase drug release in buffer medium.

D-Optimal was chosen as the approach to create the experimental design for the study as it uses a simpler model in its design, which aims to minimize the variance of factor-effect estimates to create test formulations to study to obtain an optimized formulation. Other experimental design methods such as I-Optimal are available and provide better prediction performance with their experimental designs as it aims to minimize the average variance of prediction over the region of experimentation [22]. The selection criteria of an experimental design approach depends on the approach to variances of the response surface estimators that is preferred: integral/average variance approach. However, D-Optimal is easier to operate and provides nearly as desirable an outcome in its experimental design.

The pharmacokinetic parameters $\mathrm{C}_{\max }, \mathrm{T}_{\max }, \mathrm{AUC}_{0-t}$, and $\mathrm{AUC}_{0-\infty}$ were determined for the D-Optimal design optimized capsules of LPZ given to healthy beagle dogs and were statistically compared to Gastevin ${ }^{\circledR}$ capsules as a reference (KRKA, Slovenia) using the non-compartmental method with the aid of WinNonlin 5.2 software. The analysis of variance showed that the two formulations did not demonstrate bioequivalence using a $90 \%$ confidence interval range ( 80 $120 \%$ ) for $\mathrm{C}_{\max }, \mathrm{AUC}_{0-t}$ and $\mathrm{AUC}_{0-\infty}$. However, no significant difference in $\mathrm{T}_{\max }$ was found at the 0.95 significance level using the Wilcoxon signed-rank test.

The prepared capsules containing LPZ enteric-coated pellets were stable for 18 months at room conditions and 6 months in accelerated conditions. The use of D-Optimal to experimentally design an optimal dosage form was quite satisfactory. The experimental design yielded an optimal formulation that produced similar bioequivalence to the commercially available reference lansoprazole product. The stability study results have important significance for this formulation in zone IV, which includes Vietnam as well as other countries in South-East Asia.

\section{Acknowledgements}

Appreciation and acknowledgement are given to the Vietnam Ministry of Health for their support to conduct this research.

\section{Compliance with Ethical Standards}

The study protocol was approved by the ethics committee of medicine and pharmacy research at Military Medical University (Hanoi, Vietnam).

\section{Declaration of Interest}

The authors declare that there is no conflict of interest regarding the publication of this research paper.

\section{References}

[1] Richter, J.E., Kahrilas, P.J., Sontaq, S.J., Kovacs, T.O., Huanq, B. and Pencyla, J.L. (2001) Comparing Lansoprazole and Omeprazole in Onset of Heartburn Relief: 
Results of a Randomized, Controlled Trial in Erosive Esophagitis Patients. The American Journal of Gastroenterology, 96, 3089-3098. https://doi.org/10.1111/j.1572-0241.2001.05263.x

[2] DellaGreca, M., Lesce, M.R., Previtera, L., Rubino, M., Temussi, F. and Brigante, M. (2006) Degradation of Lansoprazole and Omeprazole in the Aquatic Environment. Chemosphere, 63, 1087-1093.

[3] He, W., Tian, J., Yang, M., Fan, J., Zhang, S., Guan, P., Wang, J. and Wu, W. (2011) A Comparative Study of the Effect of Different Alkaline Stabilizers on Physicochemical Properties of Lansoprazole in Formulation. Asian Journal of Pharmaceutical Sciences, 6, 89-100.

[4] Kasture, S., Gondkar, S.B., Dareka, A.B., Priyobrata, D. and Bhambar, K.V. (2011) Enhancement of Dissolution Rate of Lansoprazole Using Liquisolid Tablet Technique. International Journal of Pharmaceutical Research, 3, 27-31.

[5] Ito, Y., Arai, H., Uchino, K., Iwasaki, K., Shibata, N. and Takada, K. (2005) Effect of Adsorbents on the Absorption of Lansoprazole with Surfactant. International Journal of Pharmaceutics, 289, 69-77

[6] Yu, M., Sun, L., Li, W., Lan, Z., Li, B., Tan, L., Li, M. and Yang, X. (2011) Investigation of Structure and Dissolution Properties of a Solid Dispersion of Lansoprazole in Polyvinylpyrrolidone. Journal of Molecular Structure, 1005, 70-77.

[7] Ghebre-Sellassie, I. and Knoch, A. (2007) Pelletization Techniques. In: Swarbrick, J., Ed., Encyclopedia of Pharmaceutical Technology, 3rd Edition, Informa Healthcare, New York, 2651-2663.

[8] Pachabhai, D., Bhama, S., Kumar, R.S. and Perumal, P. (2012) Formulation and Evaluation of Lansoprazole Enteric Coated Pellets. International Journal of Pharmaceutical Sciences and Research, 3, 1-19.

[9] Jayakar, B., Bhowmik, D., Sharma, A. and Bhowmik, D. (2010) Formulation and Evaluation of Modified Release Capsules of Lansoprazole. Pharmaceutical Research, 4, 61-73.

[10] Venkateswarlu, P. (2013) Formulation and in Vitro Evaluation of Lansoprazole Delayed Release Capsules. International Journal of Pharmaceutical Research and Innovation, 4, 328-336.

[11] The United States Pharmacopoeia XXXV (2013) Lansoprazole Delayed-Release Capsules.

[12] Aksu, B., Gokce, E.H., Rencber, S. and Ozy Azici, M. (2014) Optimization of Solid Lipid Nanoparticles Using Gene Expression Programming (GEP). Latin American Journal of Pharmacy, 33, 675-684.

[13] Costa, P. and Lobo, J.M.S. (2001) Modeling and Comparison of Dissolution Profiles. European Journal of Pharmaceutical Sciences, 13, 123-133.

[14] Bolton, S. and Bon, C. (2010) Pharmaceutical Statistics: Practical and Clinical Applications. 5th Edition, Informa Healthcare, 266-300.

[15] Chun, A.H.C., Eason, C.J., Shi, H.H. and Cavanaugh, J.H. (1995) Lansoprazole: An Alternative Method of Administration of a Capsule Dosage Formulation. Clinical Therapeutics, 17, 441-447.

[16] Song, M., Gao, X., Hang, T.J. and Wen, A.D. (2009) Pharmacokinetic Properties of Lansoprazole (30-mg Enteric-Coated Capsules) and Its Metabolites: A Single-Dose, Open-Label Study in Healthy Chinese Male Subjects. Current Therapeutic Research, 70, 228-239.

[17] Deshpande, J.V., Gupte, V.S., Kadam, V.M., Gosar, C., Deshmukh, S., Gupte, R. and Tamhankar, V. (2004) Enteric Coated Stable Oral Pharmaceutical Composition of Acid Unstable Drug and Process for Preparing the Same. United States Patent 20040028737.

[18] Singh, S.K., Borkhataria, C.H., Seth, N.R., Patel, R.P., Singh, S. and Parmar, G.R. (2009) Formulation and in Vitro Evaluation of Lansoprazole Micropellets. Interna- 
tional Journal of Pharm Tech Research, 1, 1530-1540.

[19] Rowe, R.C., Sheskey, P.J. and Quinn, M.E. (2009) Handbook of Pharmaceutical Excipients. 6th Edition, Pharmaceutical Press, 506-509.

[20] Fang, Y., Wang, G., Zhang, R., Liu, Z., Liu, Z., Wu, X. and Cao, D. (2014) Eudragit L/HPMCAS Blend Enteric-Coated Lansoprazole Pellets: Enhanced Drug Stability and Oral Bioavailability. AAPS PharmSciTech, 15, 513-521. https://doi.org/10.1208/s12249-013-0035-1

[21] Sun, W.Z., Lin, W.J. and Alai, M.S. (2012) Preparation of Microparticles for AcidLabile Lansoprazole by Solvent Evaporation Method Combined with a Spray Drying Process. Journal of Food and Drug Analysis, 20, 438-445.

[22] Bondi, R.W., Igne, B., Drennen, J.K. and Anderson, C.A. (2012) Effect of Experiment Design on the Prodiction Performance of Calibration Models Based on Near-Infrared Spectoscopy for Pharamceuitcal Application. Applied Spectroscopy, 66, 1442-1453. https://doi.org/10.1366/12-06689

Submit or recommend next manuscript to SCIRP and we will provide best service for you:

Accepting pre-submission inquiries through Email, Facebook, LinkedIn, Twitter, etc. A wide selection of journals (inclusive of 9 subjects, more than 200 journals)

Providing 24-hour high-quality service

User-friendly online submission system

Fair and swift peer-review system

Efficient typesetting and proofreading procedure

Display of the result of downloads and visits, as well as the number of cited articles

Maximum dissemination of your research work

Submit your manuscript at: http://papersubmission.scirp.org/

Or contact pp@scirp.org 